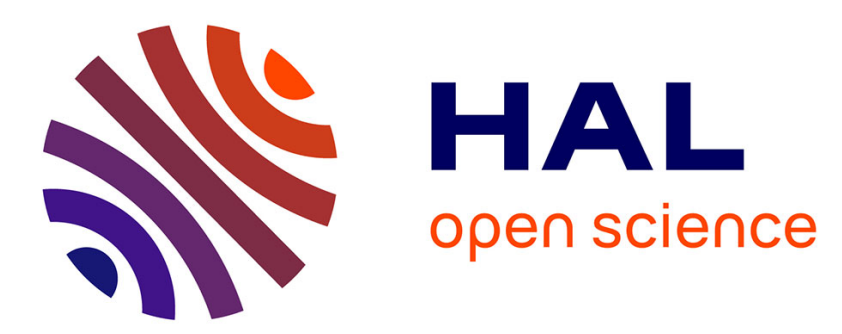

\title{
Domain duplication in magnetic tunnel junctions studied by Kerr microscopy
}

O. Lenoble, M. Hehn, D Lacour, A. Schuhl, D. Hrabovsky, J. F Bobo, Babacar Diouf, A. R Fert

\section{- To cite this version:}

O. Lenoble, M. Hehn, D Lacour, A. Schuhl, D. Hrabovsky, et al.. Domain duplication in magnetic tunnel junctions studied by Kerr microscopy. Physical Review B: Condensed Matter (1978-1997), 2001, 63 (5), 10.1103/PhysRevB.63.052409 . hal-02949497

\section{HAL Id: hal-02949497 \\ https://hal.science/hal-02949497}

Submitted on 16 Nov 2021

HAL is a multi-disciplinary open access archive for the deposit and dissemination of scientific research documents, whether they are published or not. The documents may come from teaching and research institutions in France or abroad, or from public or private research centers.
L'archive ouverte pluridisciplinaire HAL, est destinée au dépôt et à la diffusion de documents scientifiques de niveau recherche, publiés ou non, émanant des établissements d'enseignement et de recherche français ou étrangers, des laboratoires publics ou privés. 


\title{
Domain duplication in magnetic tunnel junctions studied by Kerr microscopy
}

\author{
O. Lenoble, M. Hehn, D. Lacour, and A. Schuhl \\ Laboratoire de Physique des Matériaux, UMR CNRS 7556, B.P. 239, 54506 Vandoeuvre lès Nancy Cedex, France \\ D. Hrabovsky, J. F. Bobo, B. Diouf, and A. R. Fert \\ Laboratoire de Physique de la Matière Condensée, UMR CNRS 5830 - INSA, 135 Av. de Rangueil, 31077 Toulouse Cedex, France
}

(Received 4 September 2000; published 11 January 2001)

\begin{abstract}
Magneto-optic measurements have been performed to study the magnetic response of $\mathrm{Co} / \mathrm{Al}_{2} \mathrm{O}_{3} / \mathrm{Co}$ tunnel junctions with the aim to show the domain duplication of the hard magnetic layer into the soft magnetic layer. The magnetization reversals of the hard and the soft layer have been studied separately and their ferromagnetic coupling is shown when combined in a tunnel junction. Features appearing in the variation of tunnel resistance as a function of applied field are associated to the domain phase evolution of each electrode and a deep understanding of the domain duplication is given.
\end{abstract}

DOI: $10.1103 /$ PhysRevB.63.052409

PACS number(s): 75.60.-d, 77.80.Dj, 73.40.Rw, 75.70.Pa

\section{INTRODUCTION}

Since 1995, many research programs have been devoted to the study of magnetic tunnel junctions (MTJ) because of their potential applications in data storage or signal processing. ${ }^{1,2}$ In this field area, the improvement of the tunnel magnetoresistance (TMR) signal and the control of the electrode coercive fields have focused many studies. ${ }^{2-4}$ However, the effects of the magnetic properties of the electrodes and specially their interaction on the TMR signal have been only recently the subject of development. ${ }^{5-7}$

In this last topic, we analyzed in an earlier study minor TMR cycles measured on tunnel junctions Co $(10 \mathrm{~nm}) /$ $\mathrm{AlOx} / \mathrm{Co}\left(10 \mathrm{~nm}\right.$,oxidized) ${ }^{8,9}$ This led us to introduce a domain structure duplication of the hard layer template into the soft layer via the electrode ferromagnetic coupling (see Sec. III A). Duplication deduced from TMR signal was, however, not shown in MTJ with a magnetic domain imaging method. Here, we want to illustrate the magnetic domain structure which is duplicated in the soft magnetic layer from the hard magnetic layer. We have therefore performed Kerr microscopy experiments on MTJ in order to visualize the magnetic domains. Features appearing in the variation of tunnel resistance as a function of applied field are associated to the domain phase evolution of each electrode and a deep understanding of the domain duplication is given.

\section{EXPERIMENTAL PROCEDURE}

All junctions are deposited onto float-glass substrates by sputtering of cobalt $(\mathrm{Co})$ and aluminum $(\mathrm{Al})$ targets polarized in $\mathrm{rf}$ and dc modes, respectively. The aluminum oxide barrier $(\mathrm{AlOx})$ is obtained by the oxidation of a previously deposited 1.2-nm-thick aluminum layer in an oxygen plasma in dc mode. The analyzed sample is composed of an hybrid multilayer Co $(10 \mathrm{~nm}) / \mathrm{AlOx} / \mathrm{Co}(5 \mathrm{~nm}) / \mathrm{Co}^{*}(20 \mathrm{~nm})$. The soft 10-nm-thick Co electrode has been deposited at a low argon pressure while the hard electrode is a bilayer composed by a low argon pressure deposited Co $(5 \mathrm{~nm})$ and a high argon pressure deposited Co* $(20 \mathrm{~nm})$. The easy axis of magnetization of the hard and soft layers have been cho- sen parallel. Current perpendicular to plane measurements were possible by the use of ex situ changed shadow masks that define a cross geometry of the electrodes. So, both electrodes have the same width equal to $200 \mu \mathrm{m}$ and consist of cobalt. The other experimental details have already been published. ${ }^{8}$

Kerr microscopy is based on an ellipsometric method known as "cross polarizers intensity detection." When the sample is illuminated by a polarized light, the reflected light is passing through an analyzer to transform the polarizing state to a light intensity which corresponds to the Kerr rotation. As light source is used a red electroluminescent diode $(680 \mathrm{~nm})$, its image is formed by an optical system to input focal plane of the objective to illuminate the sample by a parallel beam. The advantage of this "Kohler illumination" is that the irregularities in the brightness distribution do not cause an irregularity of the field illumination. In-plane magnetic field up to $2 \mathrm{kOe}$ in steps of $0.25 \mathrm{Oe}$ is produced by a four poles electromagnet. Images formed in the microscope are scanned with a digital charge-coupled device detector $(1000 \times 1300$ pixels $)$ at 12 bits resolution. The cross geometry of the electrodes allows one to image the domain structure of the soft or the hard electrode alone or their behavior in the junction area.

All these experiments are realized at room temperature; transport and Kerr microscopy measurements are made on the same junction.

\section{EXPERIMENTAL RESULTS}

\section{A. Transport measurements}

A typical complete TMR loop is presented in Fig. 1. It reveals clearly two resistance states even if after the reversal of the soft Co layer at $H_{0}$ a gradual increase of resistance is observed before the plateau. A minor TMR loop has been added to Fig. 1. In this case, after saturation at $500 \mathrm{Oe}$, the applied field along the easy axis is decreased down to $H_{r e v}$ and reversed again in the positive field direction. Between $H_{\text {rev }}$ and $H_{1}$, the resistance of the junction remains constant. In the positive quadrant of the loop, three resistance jumps with different signs appear at some fields named $H_{1}, H_{2}$, 


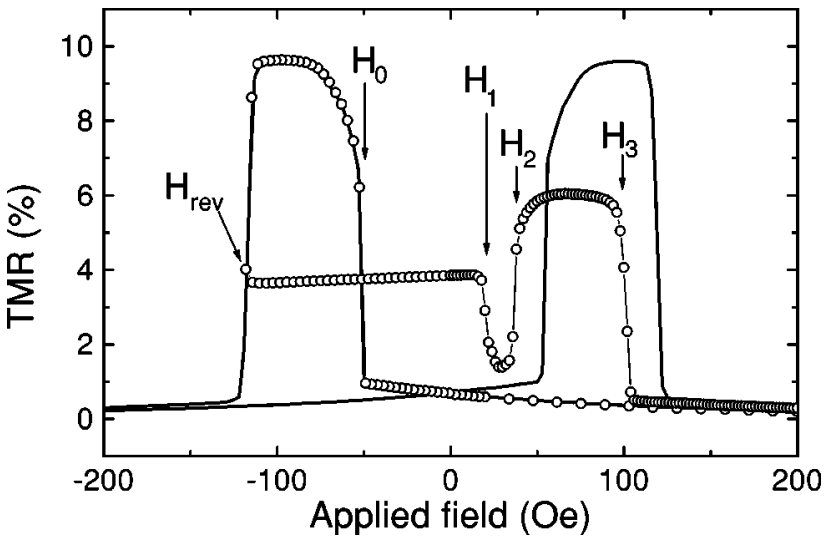

FIG. 1. Complete $(-)$ and minor $(-\bigcirc-)$ tunnel magnetoresistance loops measured on a tunnel junction Co $(10 \mathrm{~nm}) / \mathrm{Al}\left(1.2 \mathrm{~nm}\right.$, ox30 s)/Co $(5 \mathrm{~nm}) / \mathrm{Co}^{*}(20 \mathrm{~nm})$.

and $H_{3}$. Particularly interesting is the fact that for applied fields between $H_{1}$ and $H_{2}$, the resistance of the junction is close to the resistance measured when the magnetizations of the two magnetic electrodes are in a parallel configuration. Therefore, on each side of the barrier, the magnetizations are locally parallel even if the hard magnetic layer is far from magnetic saturation. As a consequence, the domain structure of the hard Co layer must be duplicated in the soft Co layer. The following aims to prove without ambiguity this interpretation.

In all following experiments, the easy axis and the applied magnetic field are horizontal and the arbitrary positive direction is to the right. Moreover, the first step consists of saturating the magnetization of the sample (electrodes or junction) with a positive applied field (images are all over bright). The numbers given below each image of Fig. 2-4 correspond to the value of the applied field at which the image is recorded. All images represent a $120 * 100$ square micrometers surface area.

\section{B. Kerr microscopy on the electrodes}

We first present the individual magnetic properties of each magnetic electrode.

The soft electrode. In this case, we have saturated the magnetization with an applied field equal to +100 Oe. Figure 2 presents three images which correspond to the reversal

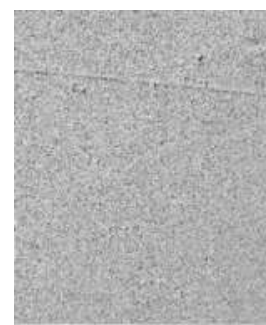

$-28 \mathrm{Oe}$

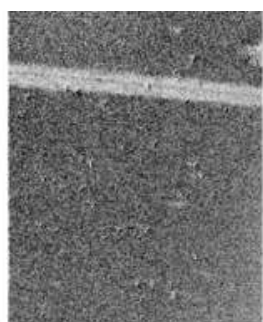

$-30 \mathrm{Oe}$

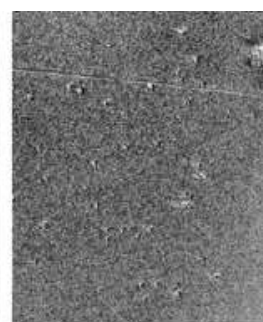

-70 Oe
FIG. 2. Kerr images showing the reversal of the soft Co electrode. The indication below each image gives the magnetic applied field at which the image was recorded.

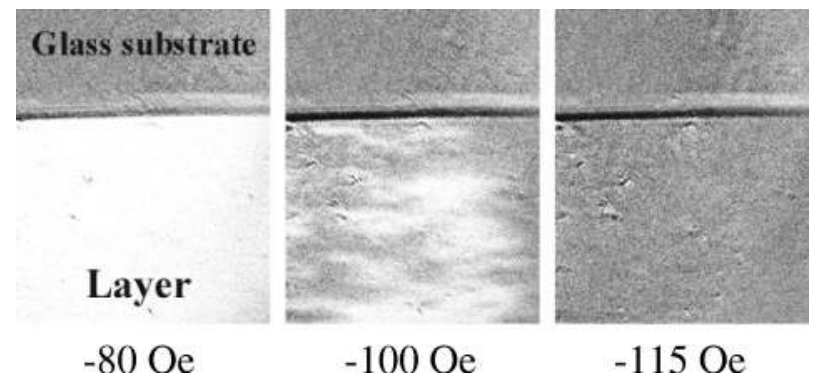

FIG. 3. Kerr images showing the reversal of the hard $\mathrm{Co} / \mathrm{Co}^{*}$ electrode. The indication below each image gives the magnetic applied field at which the image was recorded.

of the soft Co layer. This reversal is globally square with a coercive field $H_{c}$ around $30 \mathrm{Oe}$. Indeed, 2 Oe are sufficient for creating a reversed domain and for propagating it nearly all over the electrode. This is in agreement with the earlier anisotropy of magnetoresistance measurements which were indicative of a nucleation and propagation of domain walls. ${ }^{8}$ However, a close examination of the photograph recorded at -30 Oe shows a clamping of the magnetization on some defects. Indeed, scratches of the glass substrate hinder the propagation of domain walls and an increase of the applied field up to -70 Oe is then needed to saturate progressively

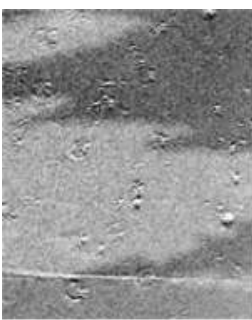

$25 \mathrm{Oe}$

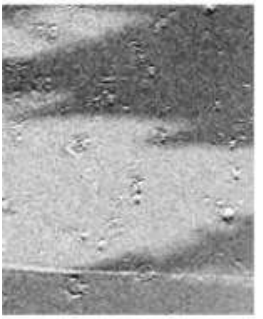

$26 \mathrm{Oe}$

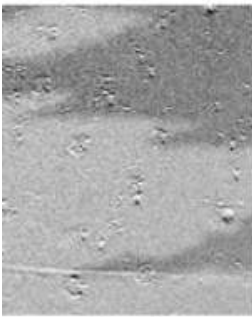

$41 \mathrm{Oe}$

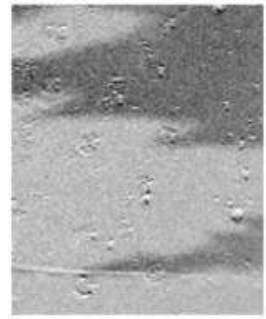

$32 \mathrm{Oe}$

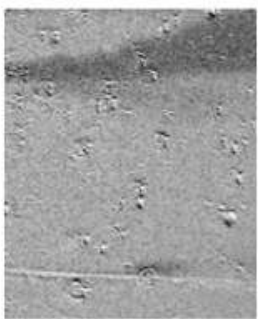

$100 \mathrm{Oe}$

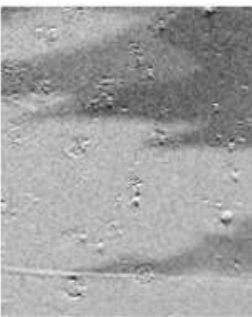

39 Oe

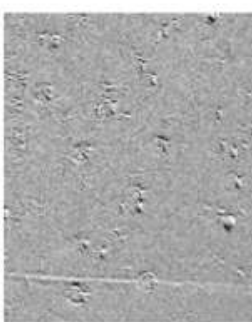

$110 \mathrm{Oe}$
FIG. 4. Kerr images showing the evolution of the magnetization in the junction (top of each image over the white line) and in the soft electrode (bottom of each image under the white line). The indication below each image gives the magnetic applied field at which the image was recorded. 
the magnetization. These results are consistent with the shape of the complete TMR loop: the jump of resistance at $H_{0}$ is attributed to the nucleation and fast propagation of domain walls; the gradual increase of resistance before reaching the highest resistance state is attributed to the progressive switching of blocked domains.

The hard electrode. In order to saturate the magnetization, we apply a magnetic field equal to +200 Oe. By decreasing this field, no evolution of the magnetic state could be observed down to -95 Oe (Fig. 3, image at -80 Oe). A further decrease initiates the reversal of the magnetization (Fig. 3, image at -100 Oe). Then one can see the formation of a domain structure composed of regions with main magnetization pointing towards the initial saturation field (bright areas) and with several tens of micrometers sized regions with switched magnetization (in dark). Between the beginning and the end of the reversal, 15 Oe are necessary to switch quite all the magnetization. An important point concerns the high stability of the domain structure. Indeed, if we stop the increase of the negative applied field before negative saturation and reverse the applied field, the domain structure does not change up to its cancellation. This point is important for the next section. As shown in Fig. 1, no variation of the tunnel resistance is observed for applied field between $H_{\text {rev }}$ and $H_{1}$ which means that the domain structures of the soft and the hard electrodes do not change.

\section{Kerr microscopy on the junction}

In the light of the magnetization reversal knowledge of each magnetic electrode, we can proceed the study with the magnetic properties of the junction in which the electrodes are strongly ferromagnetically coupled. ${ }^{8,9}$ In each photograph of Fig. 4, the upper part is the junction, i.e., the two electrodes separated by the alumina barrier and the lower part is the soft electrode taken as a reference. The two parts are separated by a white horizontal line. The sample is first saturated by applying a magnetic field of $+200 \mathrm{Oe}$. Then, it is decreased down to -100 Oe. With this field, the soft layer has totally switched (in the electrode and in the junction) and a domain structure appears in the hard layer. At this step, the applied field is increased in the positive direction and the domain structure remains unchanged up to +25 Oe. The domains which are oriented in the saturating field direction are bright while the domains which have switched appear with a dark contrast. By increasing the magnetic field, we can observe several changes in the Kerr images at some fields $H_{1,2,3}$, the same as given for Fig. 1

From 25 to $26 \mathrm{Oe}$, around $H_{1}$. In this field range, the magnetization of the soft electrode outside the junction does not change but an evolution of the contrast can be observed in the junction area: the bright domains become brighter. Indeed, the subtraction of these two images given in Fig. 5 (26-25 Oe) confirms clearly that only these regions are affected. This change is due to the switch of the soft electrode magnetization in regions located over domains in the hard electrode with magnetization oriented in the positive saturating field direction. These regions are those which experience an effective local magnetic field equal to the applied field
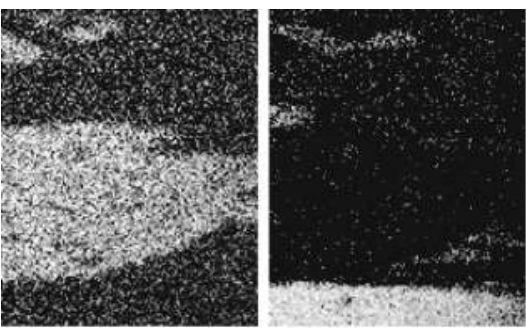

32-26 Oe

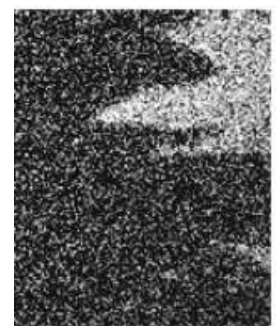

41-39 Oe

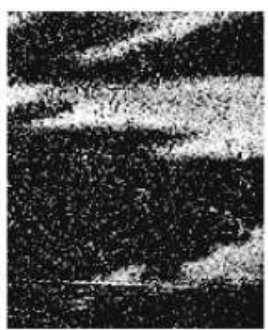

$100-41 \mathrm{Oe}$

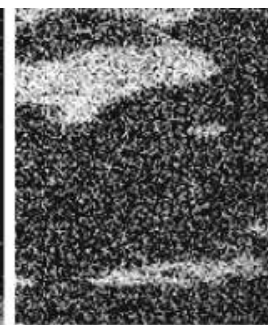

39-32 Oe

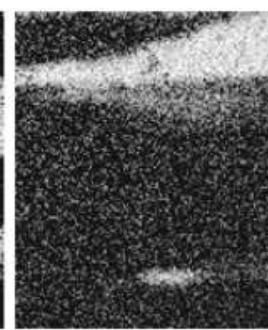

$110-100 \mathrm{Oe}$
FIG. 5. Subtractions of Kerr images of Fig. 4. The indications below each image indicate the two images of Fig. 4 which are concerned. In the bright regions, the magnetization has switched while it remains unchanged in the dark regions.

(H) plus the ferromagnetic coupling $H_{f}$ due to the bright domains in the hard electrode. So, these regions switch at a field $H_{1}$ which value is less than the intrinsic coercive field of the soft layer $H_{c}$. From those images, the nucleation process could however not be identified and specially no information on the nucleation sites could be extracted.

From 26 to $32 \mathrm{Oe}$, around $H_{c}$. In this field window, no important changes of the domain structure in the junction could be observed while the magnetization of the soft electrode outside the junction has totally switched. This reversal is illustrated by the subtraction of the images recorded at 26 and $32 \mathrm{Oe}$ (Fig. 5, 32-26 Oe). The reversal occurs at $H_{c}$ and is in agreement with the reversal presented in Fig. 2.

From 32 to $41 \mathrm{Oe}$, around $\mathrm{H}_{2}$. Since the magnetization of the soft electrode outside the junction is now oriented in the applied field direction, no evolution of the magnetic domain contrast outside the junction is expected. In the junction, the changes are confined to the dark regions of the junction which become brighter. The subtractions of these images are presented in Fig. 5 (39-32 and 41-39 Oe) to illustrate the evolution of the domain structure with the applied field. This evolution is attributed to the reversal of regions in the soft electrode which have not switched at $H_{1}$. These regions are those which experience an effective local magnetic field equal to $H$ minus the ferromagnetic coupling $H_{f}$ due to the dark domains in the hard electrode. So, these regions switch at a field $\mathrm{H}_{2}$ whose value is higher than the intrinsic coercive field of the soft layer $H_{c}$. In this case, the reversal process could be identified. Indeed, no nucleation of new domains could be observed but instead the propagation of the domains nucleated at $H_{1}$.

From 41 to $110 \mathrm{Oe}$, just before and around $\mathrm{H}_{3}$. After near saturation of the soft electrode in the junction, the main changes of contrast are easily attributed to the reversal of the 
hard layer. This is confirmed by the difference images reported in Fig. 5 (100-47 and 110-100 Oe). The progressive switching of the hard electrode domain structure proceeds by propagation and pinning of the existing domain walls.

\section{CONCLUSION}

In this paper, we have presented the visualization of the domain structure duplication from a hard magnetic layer template into a soft layer in magnetic tunnel junctions. This duplication occurs through the strong ferromagnetic coupling existing between the two magnetic electrodes. Kerr micros- copy appears to be a good tool to study these kind of effects because of its depth sensitivity (over $30 \mathrm{~nm}$ ). The results of the present study are in perfect agreement with the conclusions inferred from tunnel magnetotransport measurements.

\section{ACKNOWLEDGMENTS}

The authors thank M. Alnot, D. Basso, D. Bertrand, Ch. Féry, F. Mouginet, D. Pierre, E. Rafidison, J. P. Redoules, and S. Robert for their technical support, and P. Rottländer and J. Philbert for many fruitful discussions.
${ }^{1}$ J.S. Moodera, L.R. Kinder, T.M. Wong, and R. Meservey, Phys. Rev. Lett. 74, 3273 (1995); T. Miyazaki and N. Tezuka, J. Magn. Magn. Mater. 139, L231 (1995).

${ }^{2}$ S.S.P. Parkin et al., J. Appl. Phys. 85, 5828 (1999).

${ }^{3}$ R.C. Sousa, J.J. Sun, V. Soares, P.P. Freitas, A. Kling, M.F. da Silva, and J.C. Soares, Appl. Phys. Lett. 73, 3288 (1998).

${ }^{4}$ C. Tiusan, M. Hehn, K. Ounadjela, Y. Henry, J. Hommet, C. Meny, H.A.M. van den Berg, L. Baer, and R. Kinder, J. Appl. Phys. 85, 5276 (1999).

${ }^{5}$ L. Thomas, J. Lüning, A. Scholl, F. Nolting, S. Anders, J. Stöhr, and S.S.P. Parkin, Phys. Rev. Lett. 84, 3462 (2000); L. Thomas,
M.G. Samant, and S.S.P. Parkin, ibid. 84, 1816 (2000).

${ }^{6}$ M.R. McCartney, R.E. Dunin-Borkowski, M.R. Scheinfein, D.J. Smith, S. Gider, and S.S.P. Parkin, Science 286, 1337 (1999).

${ }^{7}$ C. Tiusan, T. Dimopoulos, K. Ounadjela, M. Hehn, H.A.M. van den Berg, V. Da Costa, and Y. Henry, Phys. Rev. B 61, 580 (2000).

${ }^{8}$ M. Hehn, O. Lenoble, D. Lacour, C. Féry, M. Piécuch, C. Tiusan, and K. Ounadjela, Phys. Rev. B 61, 11643 (2000).

${ }^{9}$ M. Hehn, O. Lenoble, D. Lacour, and A. Schuhl, Phys. Rev. B 62, 11344 (2000). 